\title{
ON A CLASS OF OPTIMIZATION PROBLEMS FOR FRAMED STRUCTURES
}

\author{
Wei H. YANG \\ Department of Applied Mechanics and Engineering Science, The University of Michigan, \\ Ann Arbor, Michigan, U.S.A.
}

Received 18 October 1977

\begin{abstract}
A unified treatment is presented for four types of problems on limit analysis of framed structures and related design problems. With the use of the lower bound theorem in plasticity these problems are formulated as standard linear programming problems. Two significant improvements are made. The new formulation of the design problems reduces the number of equations in the resulting linear program. An improved simplex algorithm for large and sparse linear programs is employed. The formulational and algorithmic improvements provide large capacity and high efficiency that are indispensable for computational analysis and design of large and complex structures.
\end{abstract}

\section{Introduction}

Engineering design usually involves a trial and error procedure that creates a prototype and reshapes it into a product based on analysis and more of ten experience. Computers now play an increasing role in engineering design. Formulation of analysis and design problems from a computational point of view falls in the area of mathematical programming.

Reviews of literature on optimal structural design were presented by Sheu and Prager [1] and Niordson and Petersen [2]. A surge of interest in optimal structural design was stimulated by the aerospace industry because weight is the most critical parameter to be minimized for a space vehicle. For earth-bound structures optimization is still an academic subject, due to the fact that the manufacturing cost of an optimal structure and the cost of design in terms of analysis and computation may offset the gain in savings of materials. Future needs for conservation and environmental protection will however make optimal structural design more compelling.

Optimal structural design problems usually assume the form of a nonlinear programming (NP) problem. Some can be transformed into or approximated by linear programs (LP) - this has the advantage of greater computational capacity and efficiency.

Optimal plastic design was first considered by Heyman [3], who used the Dines method of reducing linear inequalities - this is analogous to Gauss elimination for linear equations. Foulkes [4] gave the method a geometric interpretation and reduced the effort of searching the optimal solution to a partial exploitation of the geometric field representing the inequalities. This is conceptually parallel to Dantzig's simplex method [5] but analytically less developed. Heyman and Prager [6] presented a method of optimal plastic design of frames that is readily programmed for a computer and requires less memory than the original simplex method. Applications of mathematical programming techniques to optimal structural design are discussed by various authors in a volume edited by Pope and Schmit [7]. Continued interest in this area is reflected by a recent NATO institute on engineering plasticity by mathematical programming [8]. 
Although the possibility of reducing the optimal plastic design to a problem of linear programming has been known for some time, it has not been widely used in design practice because the optimal design of a complex structure may tax the capacity of even a large computer.

Improvements in formulation and method of solution are therefore essential. The formulation may be improved by omitting unimportant design variables in order to reduce the size of the problem. In this paper the size of the problem in terms of the number of equations is further reduced by a change of variables. A new algorithm developed by Saunders [9] for a special LP problem which involves a sparse matrix is used in this paper to enhance computational efficiency. The matrices occurring in problems of structural analysis and design are often sparse.

Four types of problems - limit analysis, optimal design, constrained design and optimal load distribution - are brought under a unified treatment. An example is given for each problem.

\section{Formulation}

Prager's plastic analysis are based on an idealized material behavior known as rigid, perfectly plastic. The rigidity condition is actually stronger than necessary for limit analysis solutions. It is sufficient to assume that the material is stiff and has an asymptotic stress behavior within a small range of strains. Thus the equilibrium equations may be approximated in the undeformed geometry. The linear equilibrium equations and a set of inequalities bounding the stresses form the necessary and sufficient conditions for the lower bound theorem of the limit analysis [10]. The perfectly plastic behavior provides bounds on the stresses. The details of the constitutive relations within the bounds are irrelevant.

In the following, four types of problems involving framed structures are formulated as linear programming problems:

(1) The limit analysis problem: Given a structure with known load-carrying capacity of each member and a set of proportional loads, find the minimum proportional factor such that the structure collapses.

(2) The optimal design problem: Given the layout of a structure and a fixed set of loads, find the distribution of material over the structure that minimizes the amount of material needed to carry the loads.

(3) The constrained design problem: Given the same conditions as in (2), find the optimization under technological constraints such as the constraint that all members must be prismatic, or that only a few types of member sizes are available.

(4) The optimal load distribution problem: Given a structure, find the optimal load distribution on the structure such that the total load is maximized. The loads can be partially fixed such as dead load, and only the remaining loads are subjected to optimal distribution.

We shall assume that all loads are in the form of concentrated forces. If distributed loads are present on a member, a discretization method may be used to replace the distributed loads by an equivalent set of concentrated loads such that the equilibrium condition is not altered beyond the member. Such a replacement will either render the collapse load lower than that for the distributed load or make the design more conservative [10]. If the discretization is refined, the solution for the replacement load approaches that for the distributed load.

Assume that the bending strength is the weakest link of the load-carrying capacity of the structural members. A member can carry the axial, shear and torsional loads automatically as long as 
the bending moments in the member are within its limit. This assumption is of course not always valid. However, such a simplified problem will help to present clearly the theme of this thesis, which will certainly be applicable when axial, shear and torsional effects are included.

The moment distribution curve for a straight member under concentrated loads is polygonal. The vertices of the polygon are at the locations where loads are applied and at the end points of the member that is connected to other parts of the structure. We shall call these points in the structure the point set of the domain. The set of values of moments defined on the domain in the form of a vector

$$
M=\left[M_{1}, M_{2}, \ldots M_{n}\right]^{\mathrm{t}}
$$

represents the moment distribution in the structure, where $n$ is the dimension of the domain set (or the total number of loads and connections). The maximum and minimum values of the moments in the structure are contained in the moment vector.

These moments $M_{i}$ must satisfy equations of equilibrium for any free-body taken from the structure. However, a free-body approach introduces other static qualities such as axial and shear forces. An alternative method of deriving the equations of equilibrium is the principle of virtual work [10]. Applying the principle to the framed structures, only moments enter the equations of equilibrium. The number of independent equations is

$$
m=n-r,
$$

where $r$ is the degree of redundancy of the structure.

The equations of equilibrium can be written in the matrix form

$$
A M=F
$$

where $\boldsymbol{F}=\left[F_{1}, F_{2}, \ldots F_{m}\right]^{\mathrm{t}}$ is a force vector containing information of the applied loads, and $\boldsymbol{A}$ is an $m \times n$ matrix whose constant coefficients contain information of the dimensions and layout of the structure.

Each component $M_{i}$ of the vector $M$ is bounded by limits

$$
-L_{i} \leqslant M_{i} \leqslant U_{i}, \quad i=1,2, \ldots n
$$

where $L_{i}$ and $U_{i}$ are positive constants known as fully plastic moments which depend on the material property and the cross-section of the member. We shall call these constants material limits. In the case of a member with homogeneous material and symmetrical cross-section, $L_{i}$ and $U_{i}$ are equal.

A solution satisfying equation (3) and the inequalities (4) is a lower bound solution for which the load is safe on the given structure or the structure is safe under the given load.

An inequality can be changed to an equation by the use of a surplus or slack variable [11]. After such changes are made, both (3) and (4) are equations and can be combined to form a linear system whose number of unknowns is always greater than the number of equations. Thus the system admits infinitely many solutions which form a convex set - a hyperpolyhedron - in the Euclidean space of the variables. Among these solutions any one that optimizes an objective func- 
tion is called the optimal solution. The optimal solution may not be unique, but the optimal value of the objective function is always unique. An objective function is derived from each of the four problems mentioned.

\subsection{Limit analysis problem}

The limit analysis of a framed structure under a proportional load $\boldsymbol{F}=f \boldsymbol{a}_{F}$, where $f$ is a scalar variablc and $a_{F}$ is a constant $m$-vcctor, has long been recognized as a standard LP problem:

$$
\max C^{\mathrm{t}}\left[\begin{array}{l}
M \\
f
\end{array}\right], \quad\left[A \mid-a_{F}\right]\left[\begin{array}{l}
M \\
f
\end{array}\right]=o_{m}, \quad-L \leqslant M \leqslant U,
$$

where $C^{\mathrm{t}}=\left[o_{n}^{\mathrm{t}}, 1\right], L=\left[L_{1}, L_{2}, \ldots L_{n}\right]^{\mathrm{t}}, U=\left[U_{1}, U_{2}, \ldots U_{n}\right]^{\mathrm{t}}$ are the given constant vectors, and $\boldsymbol{A}$ is an $m \times n$ matrix.

\subsection{Optimal design problem}

For an optimal design problem the load is specified. Hence $F$ is a constant vector. However, the material limits will now be made variables to allow an optimal material distribution that minimizes the total material needed for the structure to support the given load.

Since each $M_{i}$ cannot assume both positive and negative values at the same time, the statement $-L_{i} \leqslant M_{i} \leqslant U_{i}$ with variable limits is equivalent to

$$
\left|M_{i}\right| \leqslant B_{i}, \quad B_{i} \geqslant 0, \quad i=1,2, \ldots n,
$$

where $B_{i}$ is a variable material limit at the point $i$. This quantity $B_{i}$ depends on the yielding stress $\sigma_{Y}$ and the cross-section of the structural members in the form $B_{i}=\phi \sigma_{Y} A_{i}^{3 / 2}$, where $\phi$ is the dimensionless shape vector, and $A_{i}$ is the cross-sectional area at point $i$. If $\phi$ and $\sigma_{Y}$ are fixed, the total volume of the structural material is then a constant multiple of the integral

$$
\mathcal{S}=\int_{l} B(s)^{2 / 3} \mathrm{~d} s
$$

where $s$ is a linear variable running through the length of all members, $l$ is the total linear length of all members, and $B(s)$ is a piecewise linear function with its vertex values defined by the set $\left\{B_{i} \mid i=1,2, \ldots n\right\}$.

To stay in LP formulation, an alternative optimal design is considered here. A pseudo-volume defined by

$$
V=\int_{l} B(s) \mathrm{d} s
$$

is minimized instead. If some dimensions of the member cross-section is fixed such that $B_{i} \propto A_{i}$, then minimal pseudo-volume design is the same as minimal volume design. 
The inequality sign in (6) should be removed for the optimal design problem since each $B_{i}$ will be minimized to just large enough to carry the moment $M_{i}$, that is $B_{i}=\left|M_{i}\right|$. We can thus replace equation ( 8 ) by

$$
V=\int_{l}|M(s)| \mathrm{d} s=\sum \int_{s_{i}}^{s_{j}}|M(s)| \mathrm{d} s,
$$

where $M(s)$ is the moment function for the structure, $s_{i}$ and $s_{j}$ are two adjacent points in a member, and the summation collects the integrals between all such pairs in the structure. Since the moment distribution is polygonal, $M(s)$ is a linear function between $s_{i}$ and $s_{j}$, and the integral over the interval can be replaced by

$$
\int_{s_{i}}^{s_{j}}|M(s)| \mathrm{d} s=\frac{1}{2} l_{i j}\left(\left|M_{i}\right|+\left|M_{j}\right|\right),
$$

where $l_{i j}$ is the length of the member between $s_{i}$ and $s_{j}$. If $M(s)$ vanishes between $s_{i}$ and $s_{j}$, one additional point is needed where $M(s)=0$. This point is not known in advance but can be located iteratively.

Substituting (10) into (9), we obtain

$$
V=C^{t} M^{+}
$$

where $C$ is an $n$-dimensional vector whose $i$ th element is a weighted length associated with point $i$, and the vector $\boldsymbol{M}^{+}$is

$$
M^{+}=\left(\left|M_{1}\right|,\left|M_{2}\right|, \ldots\left|M_{n}\right|\right)^{t}
$$

Now the optimal design problem can be rewritten in the form

$$
\min C^{\mathrm{t}} M^{+}, \quad A M=F,
$$

where the objective function

$$
C^{\mathrm{t}} M^{+}=\sum_{i=1}^{n} C_{i}\left|M_{i}\right|
$$

is not a linear function in its present form. By a change of variables

$$
\left.\begin{array}{l}
M_{i}=Y_{i}-Z_{i} \\
\left|M_{i}\right|=Y_{i}+Z_{i}
\end{array}\right\} i=1,2, \ldots n,
$$


where $Y_{i}$ and $Z_{i}$ are nonnegative variables, the LP system (13) reduces to

$$
\min \left[C^{\mathrm{t}}, C^{\mathrm{t}}\right]\left[\begin{array}{l}
Y \\
Z
\end{array}\right], \quad[A \mid-A]\left[\begin{array}{c}
Y \\
Z
\end{array}\right]=F
$$

Note that the number of equations in the above LP system is only the number of the equilibrium equations. This is better than any formulation of its kind in the literature. The reduction of the number of equations is at the expense of increasing the number of variables. But a net gain is obtained since the reduced dimension of the basis of the LP system results in much less computation.

Although the objective function (which is being minimized) is the pseudo-volume rather than the true volume of the structural material, the solution which minimizes the pseudo-volume is usually close to the solution for the minimum volume design. The former can always be used as an initial trial solution in the NP formulation for the true minimum volume design.

\subsection{Constrained design problem}

We shall consider a structure with given layout and loads. In addition, we require all members to be prismatic between joints that connect the members. The joints are assumed always to be stronger than the members. Members performing a similar function are required to be identical. The problem is to determine the material limits for a few groups of members such that the total cost or volume of the structural material is minimized. We further assume $L=U=B$, where the elements of $B$ are positive but not all distinct. Let the distinct elements of $B$ be $\hat{B}_{1}, \hat{B}_{2}, \ldots \hat{B}_{j}$, where $j$ is the number of different types of members to be used in the structure. We can write

$$
B=J \hat{B}
$$

where $J$ is an $n \times j$ matrix whose elements are either 0 or 1 . An entry of 1 in $J$ equates a material limit at a point to the limit of the member containing the point. A specific $J$ is given in the next section.

The constrained design problem can be expressed in the form

$$
\min C_{B}^{\mathrm{t}} \hat{B}, \quad A M=F, \quad M^{+} \leqslant B,
$$

where $C_{B}$ is a constant vector of dimension $j$ that weights the cost or volume of different types of members used in the structure.

Using (15), (16) and (17), the LP system (18) can be manipulated into the standard form

$$
\min \left[\boldsymbol{o}_{2 n}^{\mathrm{t}}, C_{B}^{\mathrm{t}}\right]\left[\begin{array}{c}
\boldsymbol{Y} \\
\boldsymbol{Z} \\
\hat{B}
\end{array}\right], \quad\left[\begin{array}{c|c|c}
\boldsymbol{A} & -\boldsymbol{A} & \boldsymbol{O} \\
\hline \boldsymbol{I} & \boldsymbol{I} & -\boldsymbol{J}
\end{array}\right]\left[\begin{array}{c}
\boldsymbol{Y} \\
\boldsymbol{Z} \\
\hat{B}
\end{array}\right] \overline{<}\left[\begin{array}{c}
\boldsymbol{F} \\
\boldsymbol{o}_{n}
\end{array}\right],
$$

where $\boldsymbol{O}$ is an $m \times j$ null matrix, and $\boldsymbol{I}$ is an $n \times n$ identity matrix. The variables $\boldsymbol{Y}, \boldsymbol{Z}, \hat{\boldsymbol{B}}$ are nonnegative. The moment is given by $\boldsymbol{M}=\boldsymbol{Y}-\boldsymbol{Z}$. 


\subsection{Optimal load distribution problem}

Consider a completely specified structure such as that in the limit analysis problem. Some fixed loads and a family of undirectional loads are applied on the structure. We seek the optimal distribution of the family on the structure such that the sum of all loads in the family is maximized.

The LP formulation for optimal load distribution is achieved by first assigning a set of points on the structure where the family of loads shall be applied. At each point $i$ the load has an unknown magnitude $G_{i}$. The vector $G=\left[G_{1}, G_{2}, \ldots G_{k}\right]^{t}$ then represents the load distribution, where $k$ is the number of loads in the family. In addition to these $k$ points the domain set has a total number of $n$ points including the points of action of the fixed loads and the connection points of the structural members. The equilibrium equation takes the form

$$
A M=E G+F,
$$

where $\boldsymbol{A}$ and $\boldsymbol{E}$ are known matrices of dimensions $m \times n$ and $m \times k$, respectively, and $\boldsymbol{F}$ is a fixed force vector of dimension $m$.

Combining (20) with the material limits and lower bounds on the loads, we have a linear programming system for the optimal load distribution

$$
\begin{aligned}
& \max \left[\boldsymbol{o}_{n}^{\mathrm{t}}, \boldsymbol{e}_{k}^{\mathrm{t}}\right]\left[\begin{array}{l}
\boldsymbol{M} \\
\boldsymbol{G}
\end{array}\right], \quad\left[\begin{array}{ll}
\boldsymbol{A} & -\boldsymbol{E}
\end{array}\right]\left[\begin{array}{l}
\boldsymbol{M} \\
\boldsymbol{G}
\end{array}\right]=\boldsymbol{F}, \\
& -L_{i} \leqslant M_{i} \leqslant U_{i}, \quad i=1,2, \ldots n, \\
& G_{i} \geqslant 0, \quad i=1,2, \ldots k \text {, }
\end{aligned}
$$

where $e_{k}^{\mathrm{t}}=[1,1, \ldots 1]$ is the vector of dimension $k$ with all elements equal to unity.

\section{Examples}

Consider a two-story frame (fig. 1) with dimensions and loads as shown. This is an example problem used in [10]. There are 14 points in the domain set shown. The degree of redundancy of the structure is 6 , so that there are $14-6=8$ independent equations of equilibrium.

Example 1. First consider the limit analysis of the frame structure in fig. 1. Let the material limits of the lower columns and beam be $3 M_{0}$, upper columns $M_{0}$ and upper beam $2 M_{0}$, where $M_{0}$ is a constant material limit having the dimension of a moment.

Introducing the dimensionless moments and force,

$$
m_{i}=M_{i} / M_{0}, \quad i=1,2, \ldots 14, \quad f=F L / M_{0},
$$

where $L$ is the length and $F$ is the force given in fig. 1, the problem is to determine the maximum value of $f$ before the structure collapses.

The convention is used that counter-clockwise moments are positive. Eight different virtual dis- 


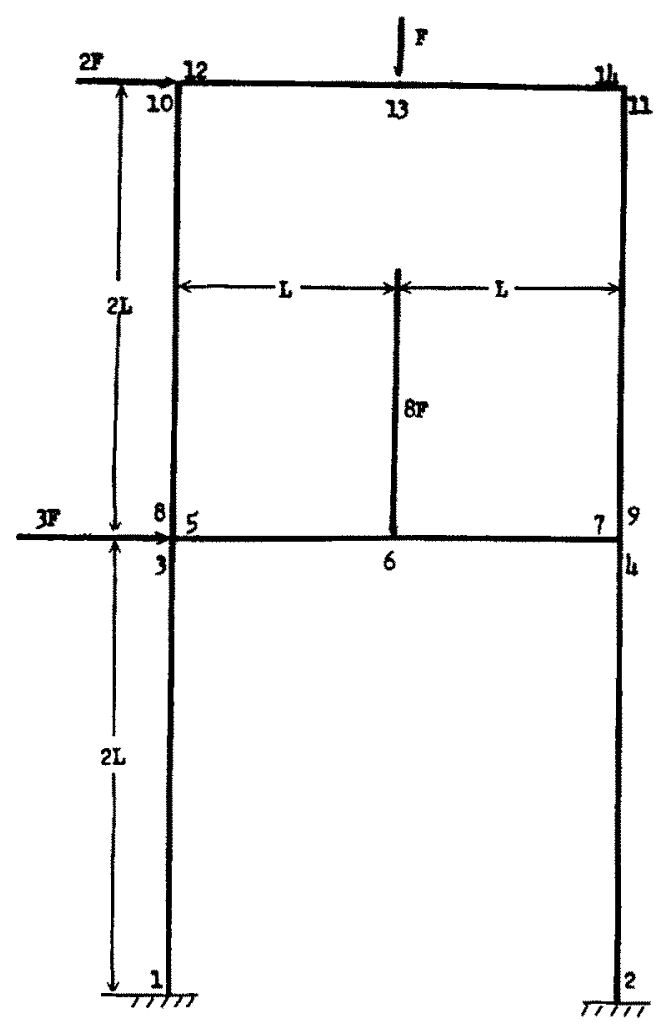

Fig. 1. A two-story frame.

placements are assigned in the forms of two panel, two beam and four joint motions; this leads to eight independent equations of equilibrium. The matrix $\boldsymbol{A}$ and the vector $\boldsymbol{a}_{F}$ are thus constructed as

$$
\boldsymbol{A}=\left[\begin{array}{rrrrrrrrrrrrrr}
1 & 1 & 1 & 1 & 0 & 0 & 0 & 0 & 0 & 0 & 0 & 0 & 0 & 0 \\
0 & 0 & 0 & 0 & 0 & 0 & 0 & 1 & 1 & 1 & 1 & 0 & 0 & 0 \\
0 & 0 & 0 & 0 & 1 & 2 & -1 & 0 & 0 & 0 & 0 & 0 & 0 & 0 \\
0 & 0 & 0 & 0 & 0 & 0 & 0 & 0 & 0 & 0 & 0 & 1 & 2 & -1 \\
0 & 0 & 1 & 0 & 1 & 0 & 0 & 1 & 0 & 0 & 0 & 0 & 0 & 0 \\
0 & 0 & 0 & 1 & 0 & 0 & i & 0 & 1 & 0 & 0 & 0 & 0 & 0 \\
0 & 0 & 0 & 0 & 0 & 0 & 0 & 0 & 0 & 1 & 0 & 1 & 0 & 0 \\
0 & 0 & 0 & 0 & 0 & 0 & 0 & 0 & 0 & 0 & 1 & 0 & 0 & 1
\end{array}\right]
$$

and

$$
a_{F}=\left[\begin{array}{llllllll}
10 & 4 & 8 & 1 & 0 & 0 & 0 & 0
\end{array}\right]^{\mathrm{t}},
$$

where the dimensionless length of each members is equal to 2 . The dimensionless material limits are 


$$
\begin{array}{ll}
-3 \leqslant m_{i} \leqslant 3, & i=1,2, \ldots 7, \\
-1 \leqslant m_{i} \leqslant 1, & i=8,9,10,11, \\
-2 \leqslant m_{i} \leqslant 2, & i=12,13,14 .
\end{array}
$$

With $C^{t}=\left[\begin{array}{lllllllllllllll}0 & 0 & 0 & 0 & 0 & 0 & 0 & 0 & 0 & 0 & 0 & 0 & 0 & 0 & 1\end{array}\right]^{t}$ the linear programming formulation of the problem is now complete. The LP algorithm provided in [9] is used on a IBM 370/91 computer to give the solution

$$
\max f=0.9090909 \text {, }
$$

which agrees with that in [10].

Example 2. Now we shall obtain the optimal design for the same example structure. At a fixed value of $f=0.9090909$ the force vector on the right-hand side of equation (16) is now

$$
F=\left[\begin{array}{l}
9.09090909 \\
3.63636364 \\
7.27272727 \\
0.90909091 \\
0.0 \\
0.0 \\
0.0 \\
0.0
\end{array}\right]
$$

The vector $C$ constructed by the trapasoidal rule is

$$
C=\left[\begin{array}{llllllllllllll}
1 & 1 & 1 & 1 & 1 / 2 & 1 & 1 / 2 & 1 & 1 & 1 & 1 & 1 / 2 & 1 & 1 / 2
\end{array}\right]^{\mathrm{t}},
$$

where the elements are the weighted dimensionless lengths of the members at all points. The optimal solution is given by

$$
\boldsymbol{M}^{+}=\left[\begin{array}{l}
5.45454545 \\
0.0 \\
0.0 \\
3.63636364 \\
0.0 \\
0.0 \\
7.27272727 \\
0.0 \\
3.63636364 \\
0.0 \\
0.0 \\
0.0 \\
0.45454545 \\
0.0
\end{array}\right]
$$




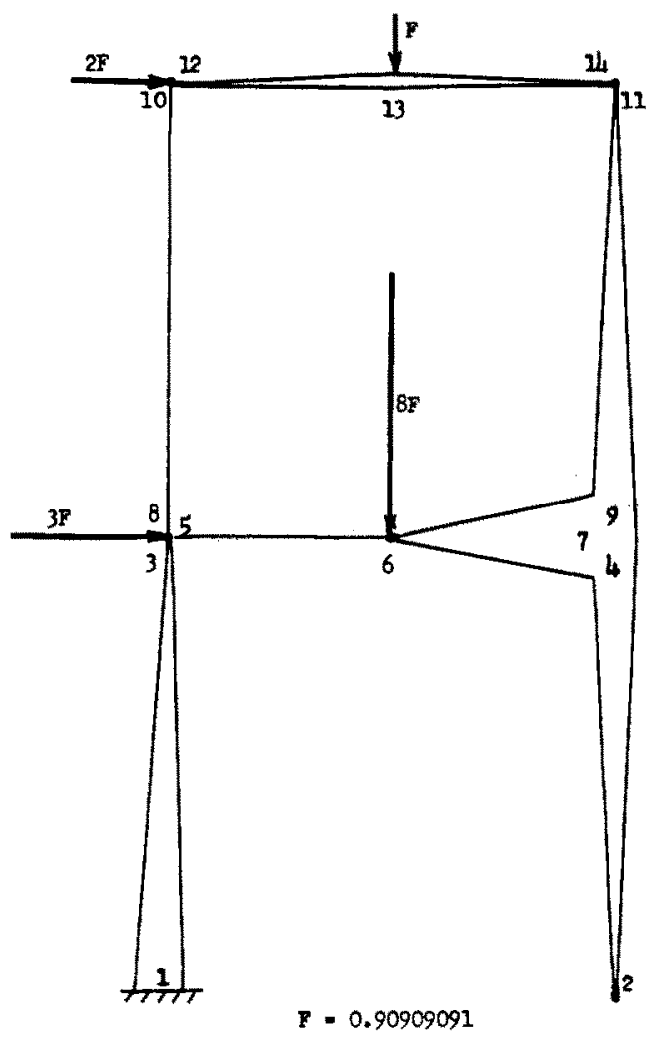

Fig. 2. Optirnal design of the two-story frame.

which indicates the magnitude of the moments as well as the material limits at every point in the domain set. Since the moment distribution is linear between two adjacent points, the structure can be illustrated with tapered members between points as shown in fig. 2 .

The beam between points 5 and 6 and the column between points 8 and 10 have zero crosssection. This is because the two members do not carry any bending moment and are therefore not needed in the model problem. But the column $8-10$ is subjected to an axial compression which is not considered in the formulation. Obviously, the inclusion of axial forces in the formulation is needed in this case. The example here still serves the purpose of demonstrating the method of solution.

The ratio of the pseudo-volume of the optimal structure to that of the original structure is

$$
V_{\text {opt }} / V_{\text {orig }}=0.64685315 \text {, }
$$

which makes a $35.3 \%$ material saving for the optimal structure.

Example 3. The third example is the constrained design of the same structure with a fixed $f=0.9090909$. We require all members to be prismatic, and only four types of members are to be used. These are lower columns, lower beam, upper columns and upper beam whose respective material limits $\hat{B}_{1}, \hat{B}_{2}, \hat{B}_{3}, \hat{B}_{4}$ are to be determined.

The matrix $J$ in its transposed form is 


$$
\boldsymbol{J}^{\mathbf{t}}=\left[\begin{array}{llllllllllllll}
1 & 1 & 1 & 1 & 0 & 0 & 0 & 0 & 0 & 0 & 0 & 0 & 0 & 0 \\
0 & 0 & 0 & 0 & 1 & 1 & 1 & 0 & 0 & 0 & 0 & 0 & 0 & 0 \\
0 & 0 & 0 & 0 & 0 & 0 & 0 & 1 & 1 & 1 & 1 & 0 & 0 & 0 \\
0 & 0 & 0 & 0 & 0 & 0 & 0 & 0 & 0 & 0 & 0 & 1 & 1 & 1
\end{array}\right]
$$

The vector $C_{B}=\left[\begin{array}{llll}4 & 2 & 4 & 2\end{array}\right]$ is obtained from the dimensionless lengths of the members. Substituting $A, J$ and $C_{B}$ into equation (19), the LP problem gives the results

$$
\hat{B}=\left[\begin{array}{l}
2.42424242 \\
2.33333333 \\
0.90909091 \\
0.90909091
\end{array}\right]
$$

which contains the material limits of the four types of members required. The ratio of the pseudovolumes between the constrained design and that of the original structure,

$$
V_{\text {cons }} / V_{\text {orig }}=0.83916084
$$

suggests a $16.1 \%$ saving from the original structure.

Example 4. Consider a framed structure with a family of 24 vertical loads and 4 horizontal loads acting at the points as shown in fig. 3 . The horizontal loads are proportional with a load factor $f$. Tables 1 and 2 show the optimal vertical load distribution for maximum total vertical load at two fixed values of $f$. The arrays of numbers represent the dimensionless vertical loads at the corresponding locations on the structure. The optimal loads on the top floor is given by the numbers from left to right in the first line of each table. The loads on the lower floors follow. We have assumed that all beam-column members of the structure have the same length and that only four types of members are used, one type for each floor. The strength of the members is arranged in increasing order from the top floor down - the material limit of the top floor members is $M_{0}=$ const; the limits for the lower floors are $2 M_{0}, 3 M_{0}$ and $4 M_{0}$. The value $f=6.8$ corresponds to the collapse under the horizontal loads alone. The tables for intermediate values of $f$ are omitted here. The total vertical load at different values of $f$ are shown in fig. 4. This is similar to the yield polygon for a two-parameter proportional loading situation.

Table 1

Vertical load distribution for $f=0$.

\begin{tabular}{rrrrrr}
\hline 6.0 & 6.0 & 6.0 & 6.0 & 6.0 & 6.0 \\
12.0 & 12.0 & 12.0 & 12.0 & 12.0 & 12.0 \\
18.0 & 18.0 & 18.0 & 18.0 & 18.0 & 18.0 \\
24.0 & 24.0 & 24.0 & 24.0 & 24.0 & 24.0 \\
\hline
\end{tabular}

Table 2

Vertical load distribution for $f=6.8$.

\begin{tabular}{rrrrrr}
\hline \multicolumn{7}{c}{ Vertical load distribution for $f=6.8}$. \\
0.0 & 6.0 & 3.0 & 6.0 & 3.0 & 6.0 \\
0.0 & 12.0 & 3.0 & 12.0 & 3.0 & 12.0 \\
0.0 & 18.0 & 3.0 & 18.0 & 3.0 & 18.0 \\
0.0 & 24.0 & 3.0 & 24.0 & 3.0 & 24.0 \\
\hline
\end{tabular}

\section{Conclusion}

Although this paper deals with a well-studied problem of limit analysis and design of framed structures, it presents a unified formulation for a class of structural optimization problems for 


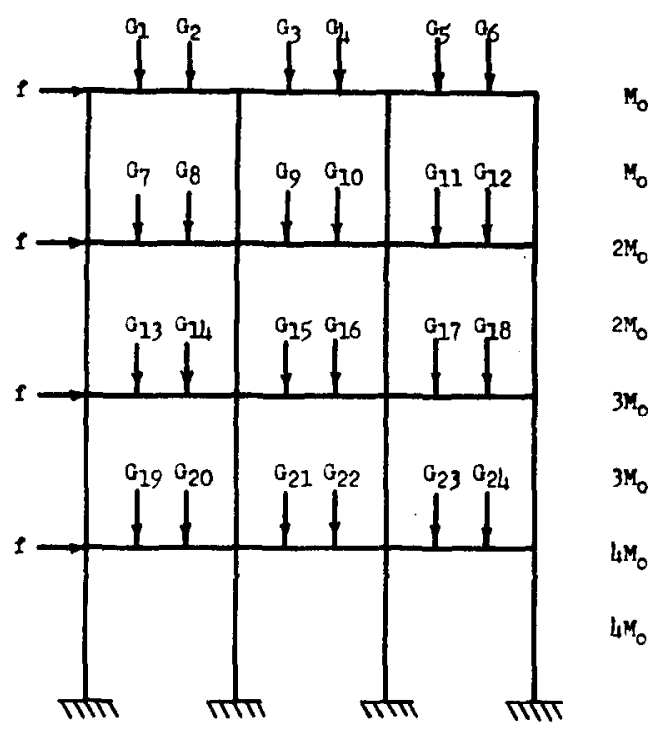

Fig. 3. Load distribution problem on a frame structure.

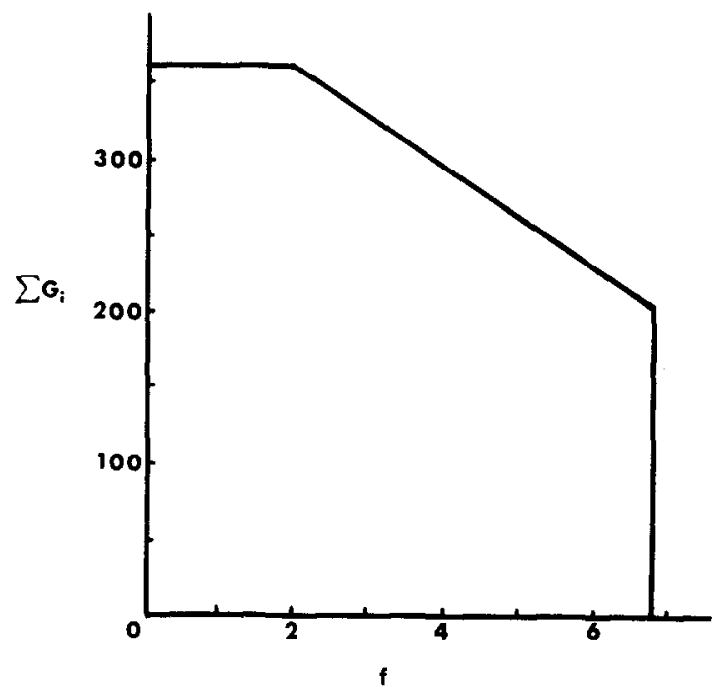

Fig. 4. Total vertical load of optimal distributions at various side loads.

which the optimal design and the optimal load distribution problems have not been previously formulated in the same manner. The new formulation in some cases requires a smaller number of equations and variables than the existing formulation. Optimal design of structures from a mechanics viewpoint alone may be regarded as being academic since labor and manufacturing costs today excced material cost. However, the constrained design and the optimal load distribution problems presented in this paper can find ready applications in conventional structural design practices and in planning the loading of an existing structure.

With the implementation of a recently developed LP algorithm the new formulation proves to be efficient and less restrictive in terms of the size of the problems that can be handled. The Saunders algorithm using updated Cholesky factors can efficiently solve LP systems up to 4000 equations with a virtually unlimited number of variables. The new algorithm, however, remains to be time-tested for various structural optimization problems. The formulation presented in this paper is readily adoptable to the standard LP routines in most computational facilities.

\section{Acknowledgment}

The author is grateful to M. Saunders and G. Golub for their generous assistance on the subject of sparse LP system and to the National Science Foundation for the NSF Grant Eng. 75-17489.

\section{References}

[1] C.Y. Sheu and W. Prager, Recent developments in optimal structure design, Appl. Mech. Rev. 21 (1968) 985 -992.

[2] F.L. Niordson and P. Petersen, A review of optimal structural design, Report No. 31 (Danish Center for Applied Mathematics and Mechanics, Tech. Univ. Denmark, 1972). 
[3] J. Heyman, Plastic design of beams and frames for minimum material consumption, Q. Appl. Math. 8 (1951) 373-381.

[4] J.D. Foulkes, Minimum weight design and the theory of plastic collapse, Q. Appl. Math. 10 (1953) 347-358.

[5] G.B. Dantzig, Linear programming and extensions (Princeton Univ. Press, Princeton, N.J., 1963).

[6] J. Heyman and W. Prager, Automatic minimum weight design of steel frames, J. Franklin Inst. 226 (1968) 339-364.

[7] G.G. Pope and L.A. Schmit, Structural design applications of MP techniques (AGARD NATO No. 149, London, 1971).

[8] NATO Advanced Study Institute on Engineering Plasticity by Mathematical Programming, Univ. Waterloo, Ontario, Canada, August 2-12, 1977.

[9] M. Saunders, A fast, stable implementation of the simplex method using Bartels-Golub updating, Symposium on Sparse matrix Computations, Argonne Natl. Lab. Sept. 9-11, 1975.

[10] P.G. Hodge Jr., Plastic analysis of structures (McGraw-Hill, New York, 1959).

[11] G. Hadley, Linear programming (Addison-Wesley, Englewood Cliffs, N.J., 1962). 